\title{
Selective ablation of nociceptive neurons for elimination of hyperalgesia and neurogenic inflammation
}

\author{
Gabriel C. Tender, M.D., Stuart Walbridge, B.S., Zoltan Olah, Ph.D., \\ Laszlo Karai, M.D., Michael Iadarola, Ph.D., Edward H. Oldfield, M.D., \\ AND RUSSELl R. LONSER, M.D.
}

Surgical Neurology Branch, National Institute of Neurological Disorders and Stroke; Pain and Neurosensory Mechanisms Branch, National Institute of Dental and Craniofacial Research, National Institutes of Health, Bethesda, Maryland; and Department of Neurosurgery, Louisiana State

University, New Orleans, Louisiana

\begin{abstract}
Object. Neuropathic pain is mediated by nociceptive neurons that selectively express the vanilloid receptor 1 (VR1). Resiniferatoxin (RTX) is an excitotoxic VR1 agonist that causes destruction of VR1-positive neurons. To determine whether RTX can be used to ablate VR1-positive neurons selectively and to eliminate hyperalgesia and neurogenic inflammation without affecting tactile sensation and motor function, the authors infused it unilaterally into the trigeminal ganglia in Rhesus monkeys.

Methods. Either RTX (three animals) or vehicle (one animal) was directly infused (20 $\mu$ l) into the right trigeminal ganglion in Rhesus monkeys. Animals were tested postoperatively at 1, 4, and 7 weeks thereafter for touch and pain perception in the trigeminal distribution (application of saline and capsaicin to the cornea). The number of eye blinks, eye wipes, and duration of squinting were recorded. Neurogenic inflammation was tested using capsaicin cream. Animals were killed 4 (one monkey) and 12 (three monkeys) weeks postinfusion. Histological and immunohistochemical analyses were performed.

Throughout the duration of the study, response to high-intensity pain stimulation (capsaicin) was selectively and significantly reduced $(\mathrm{p}<0.001$, RTX-treated compared with vehicle-treated eye [mean \pm standard deviation]): blinks, $25.7 \pm 4.4$ compared with $106.6 \pm 20.8$; eye wipes, $1.4 \pm 0.8$ compared with $19.3 \pm 2.5$; and squinting, $1.4 \pm 0.6$ seconds compared with $11.4 \pm 1.6$ seconds. Normal response to sensation was maintained. Animals showed no neurological deficit or sign of toxicity. Neurogenic inflammation was blocked on the RTX-treated side. Immunohistochemical analysis of the RTX-treated ganglia showed selective elimination of VR1-positive neurons.

Conclusions. Nociceptive neurons can be selectively ablated by intraganglionic RTX infusion, resulting in the elimination of high-intensity pain perception and neurogenic inflammation while maintaining normal sensation and motor function. Analysis of these findings indicated that intraganglionic RTX infusion may provide a new treatment for pain syndromes such as trigeminal neuralgia as well as others.
\end{abstract}

\author{
KEY WORDS • neuropathic pain \\ trigeminal ganglion $\bullet$ treatment
}

New treatment approaches directed at mechanisms of neuropathic pain have led to the description of the VR $1,{ }^{2}$ a molecular integrator for pain. ${ }^{8}$ Nociceptive (Type 2 A-delta fiber and $\mathrm{C}$ fiber) neurons expressing this receptor mediate hyperalgesia and neuropathic pain. ${ }^{5,7}$ Resiniferatoxin is a VR1 agonist that induces calcium-mediated cell death within minutes of binding to VR1-expressing neurons. ${ }^{6}$ Because VR1 is expressed only by this subset of nociceptive neurons, targeting and RTX-induced ablation of these cells may permit selective elimination of pathological pain ${ }^{4}$ while preserving tactile sensation (A-alpha, A-beta, and Type 1 A-delta fiber neurons) and motor neurons. To determine if this unique selective toxicity for VR1-positive

Abbreviations used in this paper: $\mathrm{PBS}=$ phosphate-buffered saline; $\mathrm{RTX}=$ resiniferatoxin; $\mathrm{SD}=$ standard deviation; VR1 = vanilloid receptor 1 . neurons can be used to eliminate hyperalgesia without affecting normal tactile sensation and motor function, we infused RTX unilaterally in nonhuman primate trigeminal ganglia.

\section{Materials and Methods}

\section{Survival Surgery}

Four adult Rhesus monkeys underwent intraganglionic infusion of RTX (three animals) or vehicle (one animal) via a right middle fossa surgical approach. General anesthesia was induced in the animals and they were intubated. The temporal scalp was incised in a $\mathrm{U}$-shaped fashion, and a myocutaneous flap was based over the pinna of the ear. A craniectomy was centered over the external auditory canal. 
The dura mater was elevated off the skull base. Through direct visualization, a blunt 32-gauge needle was introduced 3 to $4 \mathrm{~mm}$ into the trigeminal ganglion. Convective infu$\operatorname{sion}^{1}(4 \mu \mathrm{l} /$ minute) of $20 \mu \mathrm{l}$ RTX solution $(0.1 \mathrm{mg} / \mathrm{ml}$ concentration containing Tween- 80 and $0.05 \%$ ascorbic acid in PBS) or vehicle was performed. After completing the infusion, the needle was removed and the musculocutaneous flap was closed in layers. The animal was awakened and extubated. All animals were observed twice daily for medical problems or neurological deficits throughout the study period (up to 3 months).

\section{Sensory Testing}

A short-term survival (4 weeks) animal (one experimental monkey) was tested at 1 week postoperatively. Longterm survival (12 weeks) animals (two experimental monkeys and one control monkey) were tested at 1, 4, and 7 weeks postoperatively. The animals were transferred from their housing cage to a transparent observation cage where they could be observed from various angles by multiple observers for accurate data accrual (that is, number of blinks and eye wipes and duration of squinting).

All animals underwent specific testing of trigeminal ganglion- mediated corneal tactile sensation and hyperalgesia. Normal corneal sensation was assessed after the animal had been momentarily immobilized in the observation cage and normal saline $(0.5 \mathrm{ml})$ at room temperature $\left(18^{\circ} \mathrm{C}\right)$ applied to the right eye. Immediately after saline application, the animal was released and the number of blinks during a 3minute period was recorded. Testing was conducted in an identical manner in the left eye. Corneal hyperalgesia was assessed after the animal had been momentarily immobilized in the observation cage and capsaicin $(0.01 \% ; 0.5 \mathrm{ml})$ applied to the right eye. The number of blinks and eye wipes and the duration of squinting (in seconds) during a 3minute period were recorded. Testing was performed in an identical manner in the left eye.

\section{Neurogenic Inflammation Testing}

Immediately before killing the animals, they were tested for neurogenic inflammation. With the monkeys in a state of anesthesia, all facial hair was removed using Nair (Carter Products, New York, NY). Capsaicin cream (3\%) was applied to the entire scalp and face. Evans blue solution $(0.1 \%$ in PBS, $1 \mathrm{mg} / \mathrm{kg}$ ) was administered intravenously 10 minutes after applying the capsaicin cream. Extravasation of the Evans blue-indicating nociceptive neuron-mediated neurogenic inflammation-was recorded photographically.

\section{Gross and Histological Analyses}

Animals were killed using an intravenous overdose of pentobarbital $(90 \mathrm{mg} / \mathrm{kg}$ ) at either 4 (one monkey) or 12 (three monkeys) weeks. All animals were perfused with PBS followed by $4 \%$ paraformaldehyde. The eyes and trigeminal ganglia were removed bilaterally and placed in $4 \%$ paraformaldehyde. Five-micron-thick axial sections throughout the entire trigeminal ganglion were cut on a cryostat after fixation in paraffin. These sections were stained with $\mathrm{H} \& \mathrm{E}$ or used for immunohistochemical analysis.

\section{Immunohistochemical Analysis}

The VR1-positive cells in the trigeminal ganglion were identified by immunohistochemical staining by using human anti-VR1 antibody (titer 1:500; Affinity Bioreagents, Golden, CO). The number of VR1-positive cells was determined for the bilateral trigeminal ganglia. Cell counts of VR1-positive cells were obtained from 10 randomly chosen high-power fields from the trigeminal ganglion on each side.

\section{Statistical Analysis}

Statistical analysis was performed using commercially available software and the Student paired t-test. A probability value less than 0.05 was statistically significant.

\section{Results}

\section{Sensory Testing}

Normal Tactile Sensation. Consistent with preservation of tactile sensation, there was no difference in response to corneal saline application, as determined by the number of blinks on the RTX-, vehicle-, or untreated sides (mean number of blinks $\pm \mathrm{SD}$ : $18.8 \pm 2.3,18.0 \pm 2.6$, and $18.4 \pm 3.3$, respectively; $p>0.05$; Fig. 1a).

Hyperalgesia. Consistent with the elimination of nociceptive neuron-mediated hyperalgesia, there was a dramatic reduction in the number of blinks following corneal application of capsaicin on the side of the RTX-treated ganglion compared with the vehicle- and untreated sides (mean number of blinks: $25.7 \pm 4.4$ compared with $106.6 \pm 20.8$ and $112.8 \pm 19.7$, respectively; $p<0.001$; Fig. 1b). This effect was substantiated by the significant reduction in the number of eye wipes on the side of the RTX-treated ganglion compared with the vehicle- and untreated sides (mean number of eye wipes: $1.4 \pm 0.8$ compared with $19.3 \pm 2.5$ and $16.7 \pm 2.7$, respectively; $p<0.001$; Fig. 1c). A significant reduction in the duration of squinting further supported the decreased hyperalgesic response on the side of the RTX-treated ganglion compared with the vehicle- and untreated sides (mean duration of squinting: $1.4 \pm 0.6 \mathrm{sec}-$ onds compared with $11.4 \pm 1.6$ seconds and $14.8 \pm 1.7$ seconds, respectively; $p<0.01$; Fig. 1d). These effects were durable and sustained throughout the postoperative period ( $\mathrm{p}<0.01 ;$ Fig. 1e).

\section{Clinical Evaluation}

All animals were ambulatory within 4 hours and resumed normal feeding habits within 12 hours of surgery. None had any neurological deficit or other clinical sign of toxicity throughout the duration of observation (up to 3 months). Specifically, mastication was not affected, and animals did not experience weight loss during the study period. Symmetrical and appropriate eye blinking was preserved in all animals.

\section{Neurogenic Inflammation}

Because activation of nociceptive neurons leads to the release of neurotransmitters (substance P, calcitonin generelated peptide, and adenosine 5 '-triphosphate), which promote inflammation by the release of mediators (bradykinin, serotonin, protons, and others) from neighboring nonneuronal cells and vascular tissue and thus resulting in neuro- 
a

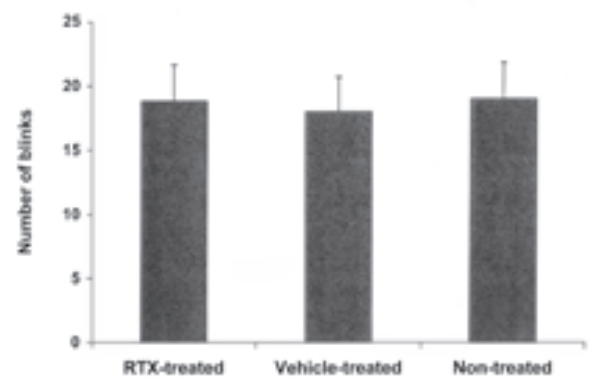

C

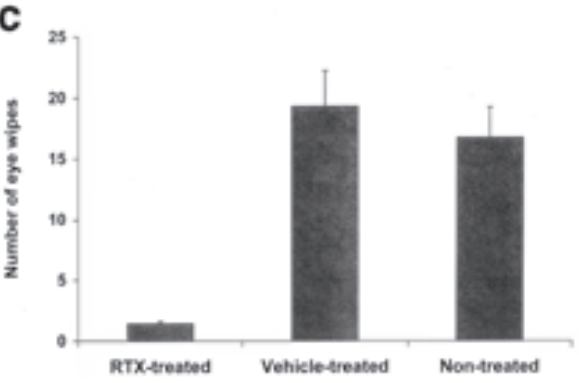

e

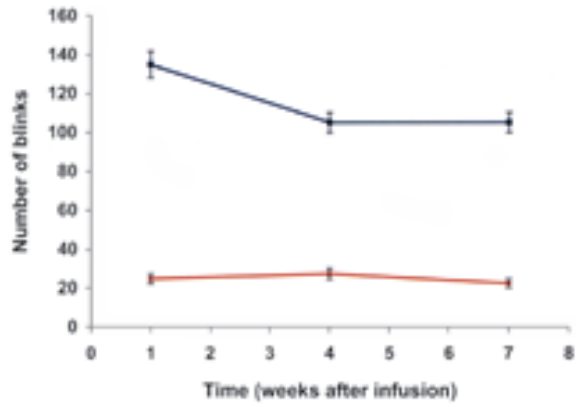

b

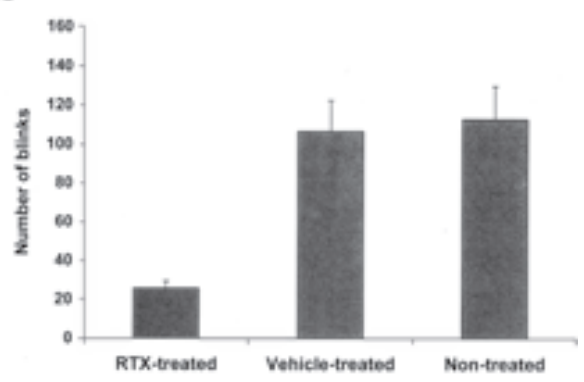

d

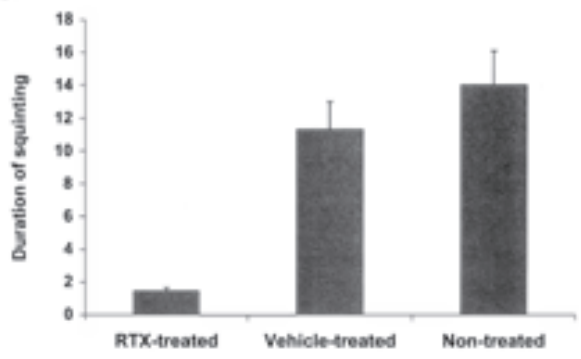

f

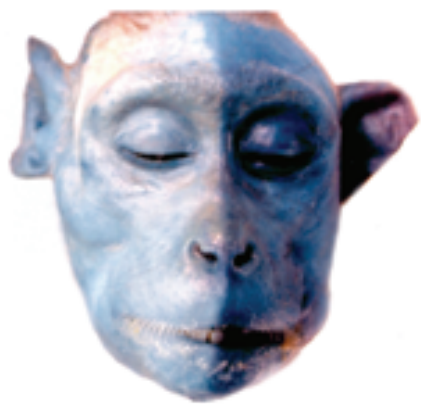

FIG. 1. Sensory and neurogenic inflammation testing. a: Bar graph demonstrating no difference in the mean (all testing) number of blinks during 3 minutes after corneal application of saline on the RTX-, vehicle-, and untreated sides ( $p>0.05$; error bars represent the SD). Corneal hyperalgesia was blocked on the RTX-treated side, as demonstrated by a significant reduction $(\mathrm{p}<0.001)$ in the mean number of blinks (b), number of eye wipes (c), and duration of squinting (in seconds, d) during 3 minutes following application of capsaicin. e: Graph depicting that the elimination of hyperalgesic sensation on the RTX-treated side (red line) compared with the untreated side (blue line) was durable over the study period (two animals; mean blinks after capsaicin application, $\mathrm{p}<0.001$ ). f: Photograph of monkey with Evans blue extravasation (blue) demonstrating that nociceptive neuron-mediated neurogenic inflammation was blocked on the RTX-treated side (right side) of the face and scalp (white).

genic inflammation, we tested the animals to see if this phenomenon was blocked by RTX. To elicit this response over the entire trigeminal-innervated territory, we applied capsaicin $(3 \%)$ to the scalp and face of control (one monkey) and experimental (three monkeys) animals just before they were killed (12 weeks posttreatment). A cutaneous inflammatory response occurred within 10 minutes of application, as evidenced by reddening of the entire face and scalp in the control animal and selective reddening of the hemiface and scalp on the untreated side in the experimental animals. Intravenous injection of Evans blue $(1 \mathrm{mg} / \mathrm{kg})$ confirmed and further delineated the regions of neurogenic inflammation by extravasating into the inflamed areas (Fig. 1f).

\section{Gross and Histological Findings}

Gross and histological examination of corneas revealed no evidence of keratitis or abrasions. Examination of the oral mucosa showed no sign of injury. Analysis of histological results revealed preservation of the normal architecture of the RTX-treated trigeminal ganglia and the absence of nonspecific toxicity.

\section{Immunohistochemical Analysis}

Immunohistochemical staining for VR1 (Fig. 2a and b) revealed uniform ablation of VR1-positive neurons (mean decrease $79.3 \pm 2.8 \%$ ) in RTX-treated ganglia compared with vehicle- and untreated ganglia (mean number of VR1positive cells per high-power field $7.3 \pm 1.6$ compared with $38.3 \pm 6.8$ and $40.6 \pm 6.1$, respectively; $p<0.001$; Fig. 2c). There was no difference between vehicle- and untreated ganglia in the control animal $(\mathrm{p}>0.05)$. 

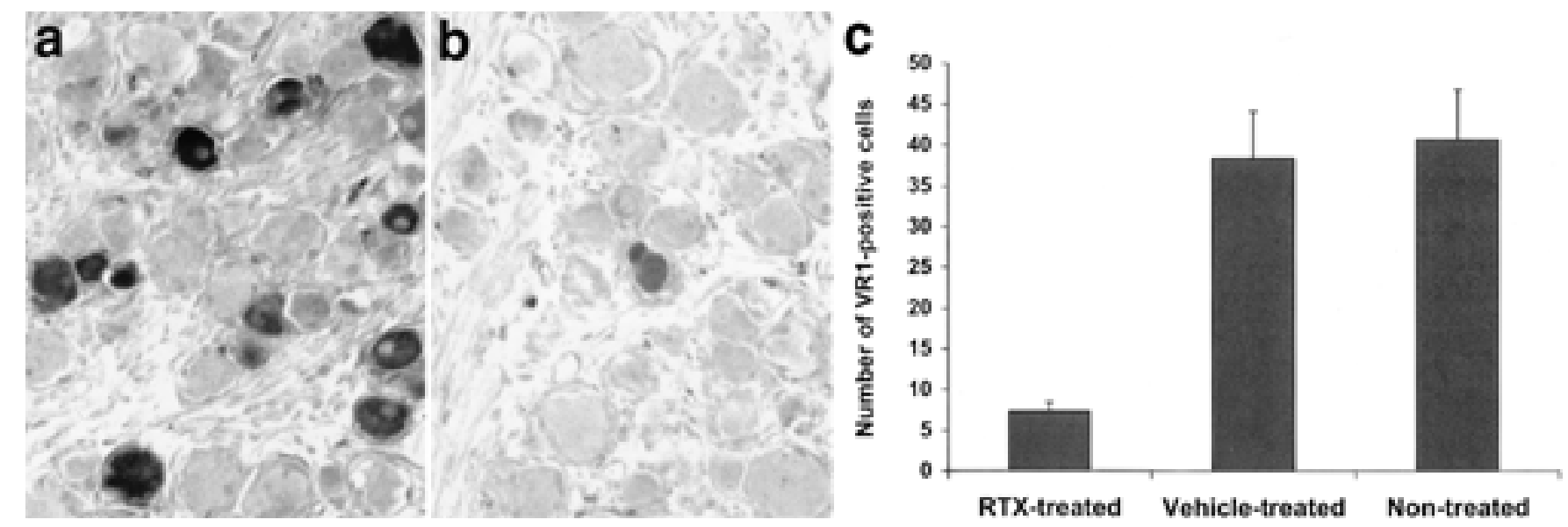

FIG. 2. Photomicrographs of tissue sections from untreated (a) and RTX-treated (b) trigeminal ganglia showing selective ablation of VR1positive neurons. c: Bar graph depicting that the mean number of VR1-positive cells per high-power field was reduced in RTX-treated ganglia compared with that in untreated ganglia (two monkeys; $\mathrm{p}<0.001$; error bars represent SD).

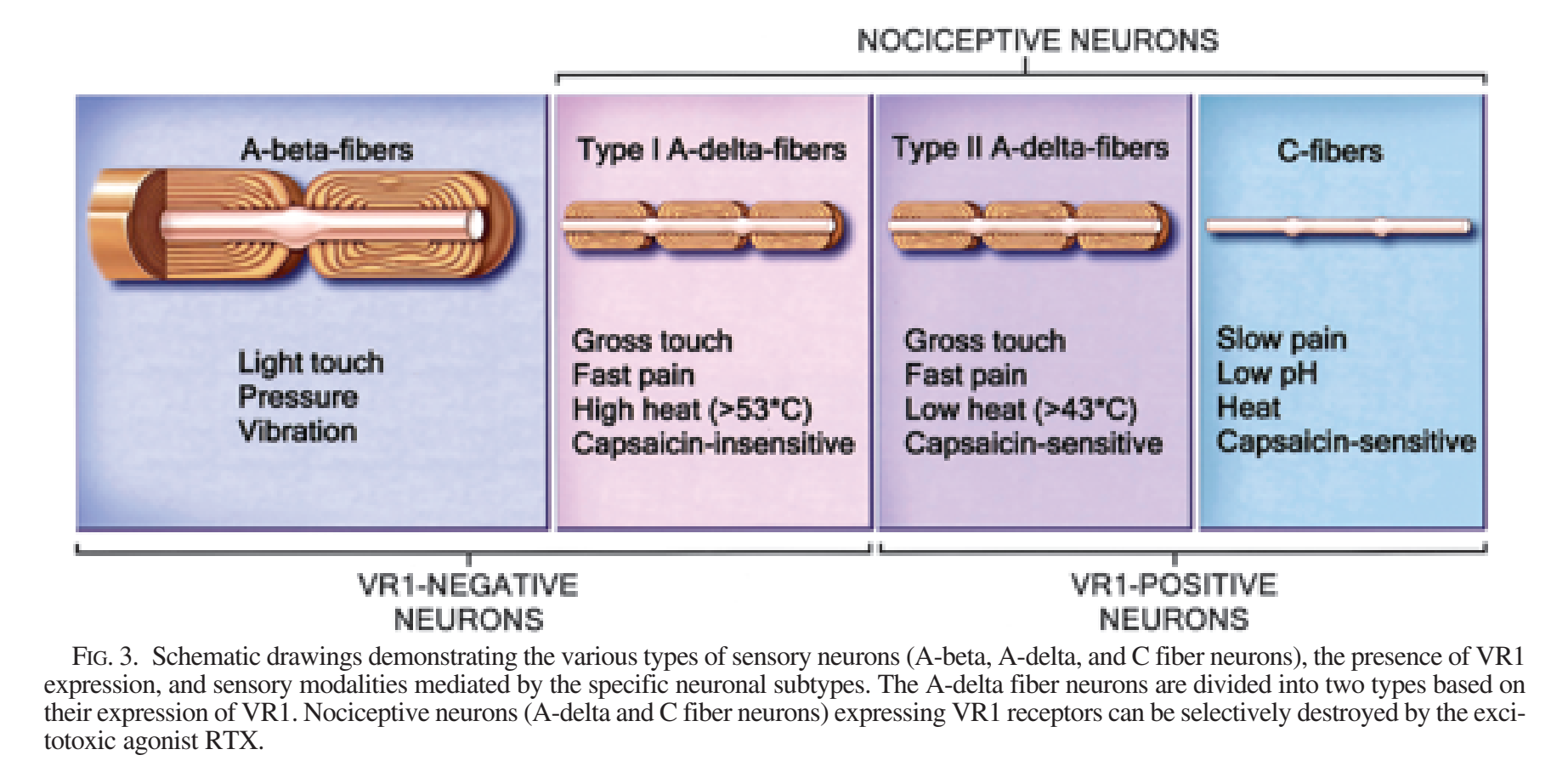

\section{Discussion}

Sensory neurons can be divided into 4 types: A-alpha fiber, A-beta fiber, A-delta fiber, and $\mathrm{C}$ fiber neurons. ${ }^{3}$ The A-alpha and A-beta fiber neurons mediate normal sensation (for example, proprioception and light touch), whereas A-delta and $\mathrm{C}$ fiber neurons primarily mediate noxious stimuli and are defined as nociceptive neurons (Fig. 3). Based on electrophysiological characteristics, the A-delta fiber neurons are subdivided into 2 types: ${ }^{5}$ Type 1 neurons do not express VR1 and have a high threshold for heat activation, whereas Type 2 neurons express VR1 and have a low threshold for heat activation. Because $\mathrm{C}$ fiber and Type 2 A-delta fiber neurons express VR1, they are selectively ablated by intraganglionic RTX infusion, which eliminates hyperalgesia, neurogenic inflammation, and the cel- lular mediators of neuropathic pain. Because A-beta fiber and Type 1 A-delta fiber neurons do not express VR1, they are not destroyed by RTX infusion, and normal tactile sensation, perception of noxious heat, and acute pain sensation are preserved.

\section{Conclusions}

Results in this study demonstrate that perfusion of trigeminal ganglia with RTX effectively and selectively ablates VR1-positive nociceptive neurons and eliminates hyperalgesia and neurogenic inflammation while maintaining normal tactile sensation and motor function. Thus, intraganglionic perfusion with RTX may provide a new sitespecific, physiological-based treatment for pain syndromes, including trigeminal neuralgia. 


\section{References}

1. Bobo RH, Laske DW, Akbasak A, Morrison PF, Dedrick RL, Oldfield EH: Convection-enhanced delivery of macromolecules in the brain. Proc Natl Acad Sci USA 91:2076-2080, 1994

2. Caterina MJ, Schumacher MA, Tominaga M, Rosen TA, Levine JD, Julius D: The capsaicin receptor: a heat-activated ion channel in the pain pathway. Nature 389:816-824, 1997

3. Julius D, Basbaum AI: Molecular mechanisms of nociception. Nature 413:203-210, 2001

4. Karai L, Brown DC, Mannes AJ, Connelly ST, Brown J, Gandal M, et al: Deletion of vanilloid receptor 1-expressing primary afferent neurons for pain control. J Clin Invest 113: 1344-1352, 2004

5. Nagy I, Rang H: Noxious heat activates all capsaicin-sensitive and also a sub-population of capsaicin-insensitive dorsal root ganglion neurons. Neuroscience 88:995-997, 1999

6. Olah Z, Szabo T, Karai L, Hough C, Fields RD, Caudle RM, et al: Ligand-induced dynamic membrane changes and cell de- letion conferred by vanilloid receptor 1. J Biol Chem 276: 11021-11030, 2001

7. Szallasi A, Blumberg PM: Resiniferatoxin, a phorbol-related diterpene, acts as an ultrapotent analog of capsaicin, the irritant constituent in red pepper. Neuroscience 30:515-520, 1989

8. Tominaga M, Caterina MJ, Malmberg AB, Rosen TA, Gilbert $\mathrm{H}$, Skinner K, et al: The cloned capsaicin receptor integrates multiple pain-producing stimuli. Neuron 21:531-543, 1998

Manuscript received June 14, 2004.

Accepted in final form October 22, 2004.

This manuscript was published in J Neurosurg 102:522-525, 2005.

Address reprint requests to: Russell R. Lonser, M.D., Surgical Neurology Branch, National Institute of Neurological Disorders and Stroke, National Institutes of Health, 10 Center Drive, Building 10, Room 5D37, Bethesda, Maryland 20892-1414. email: lonserr@ ninds.nih.gov. 
Authors 